\title{
A FIXED POINT THEOREM IN NON-ARCHIMEDEAN VECTOR SPACES
}

\author{
C. PETALAS AND T. VIDALIS
}

(Communicated by Palle E. T. Jorgensen)

\begin{abstract}
A mapping $T$ defined on a normed linear space $X$ and taking values in $X$ is said to be contractive (nonexpansive) if whenever $x$ and $y$ are distinct points in $X,\|T x-T y\|<\|x-y\|(\|T x-T y\| \leq\|x-y\|)$. In this paper we prove that every contractive mapping on a spherically complete non-Archimedean normed space has a unique fixed point.
\end{abstract}

\section{INTRODUCTION}

A non-Archimedean normed space $(X,\|\|)$ is said to be spherically complete if every shrinking collection of balls in $X$ has a nonempty intersection. If $T: X \rightarrow X$, then $T$ is said to be contractive (nonexpansive) if whenever $x$ and $y$ are distinct points in $X$,

$$
\|T x-T y\|<\|x-y\| \quad(\|T x-T y\| \leq\|x-y\|) .
$$

For the Euclidean space $\mathbb{R}$ with its usual metric, it is known that a contractive mapping $f: \mathbb{R} \rightarrow \mathbb{R}$ need not have a fixed point, e.g., the mapping

$$
f(x)=x+\frac{1}{1+e^{x}}, \quad x \in \mathbb{R} .
$$

In this paper we prove that every contractive mapping $T: X \rightarrow X$, where $X$ is a spherically complete non-Archimedean normed space, has a unique fixed point. We give also examples to show that this assertion cannot be extended to include either nonexpansive mappings or nonspherically complete spaces.

It is an open problem if a nonexpansive mapping on a reflexive normed space from a convex closed and bounded set into itself has a fixed point [13]. From our examples it follows that an analogous problem for the case of non-Archimedean normed spaces has a negative answer.

More information about non-Archimedean normed spaces can be found in [4].

\section{MAIN ReSUlt}

Theorem 1. Let $X$ be a non-Archimedean spherically complete normed space. If $T: X \rightarrow X$ is a contractive mapping then $T$ has a unique fixed point.

Received by the editors July 9, 1991 and, in revised form, October 28, 1991.

1991 Mathematics Subject Classification. Primary 46P05, 47H09, 47H99.

Key words and phrases. Fixed point, non-Archimedean vector space. 
Proof. Let $B_{a}=: B[a,\|a-T a\|]$ denote the closed spheres centered at $a$ with radii $\|a-T a\|$, and let $\mathscr{A}$ be the collection of these spheres for all $a \in X$. The relation

$$
B_{a} \leq B_{b} \quad \text { iff } \quad B_{b} \subseteq B_{a}
$$

is a partial order. Consider a totally ordered subfamily $\mathscr{A}_{1}$ of $\mathscr{A}$. From the spherical completeness of $X$, we have

$$
\bigcap_{B_{a} \in \mathscr{A}_{1}} B_{a}=: B \neq \varnothing .
$$

Let $b \in B$ and $B_{a} \in \mathscr{A}_{1}$. Then if $x \in B_{b}$,

$$
\|x-a\| \leq \max \{\|a-b\|,\|b-x\|\} \leq\|a-T a\|,
$$

since $\|a-b\| \leq\|a-T a\|$, and

$$
\|x-b\| \leq\|b-T b\| \leq \max \{\|b-a\|,\|a-T a\|,\|T a-T b\|\}=\|a-T a\| .
$$

So $x \in B_{a}$ and $B_{b} \subseteq B_{a}$ for every $B_{a} \in \mathscr{A}_{1}$. Thus $B_{b}$ is an upper bound in $\mathscr{A}$ for the family $\mathscr{A}_{1}$. By Zorn's lemma, $\mathscr{A}$ has a maximal element, say $B_{z}$, for some $z \in Z$. We claim that $z=T z$.

Suppose $z \neq T z$. Since

$$
\left\|T z-T^{2} z\right\|<\|z-T z\| \quad\left(T^{2}=: T \circ T\right)
$$

and

$$
T z \in B\left[T z,\left\|T z-T^{2} z\right\|\right] \cap B[z,\|z-T z\|],
$$

we have $B_{T z} \subseteq B_{z}$. But $z \notin B_{T z}$ by $(*)$, so $B_{T z} \subsetneq B_{z}$, and this contradicts the maximality of $B_{z}$. Therefore $T$ has a fixed point, obviously unique.

\section{NONEXPANSIVE MAPPINGS}

Theorem 2. Suppose $X$ is a spherically complete non-Archimedean normed space and $T: X \rightarrow X$ is a nonexpansive map.

Then either $T$ has at least one fuxed point or there exists a sphere $B$ of radius $r>0$ such that $T: B \rightarrow B$ and for which $\|b-T b\|=r$ for each $b \in B$.

Proof. Defining $B_{a}$ and $\mathscr{A}$ as in the proof of Theorem 1, we find similarly a maximal element $B_{z}$ of $\mathscr{A}$. For any $b \in B_{z}$ we have

$$
\|b-T b\| \leq \max \{\|b-z\|,\|z-T z\|,\|T z-T b\|\}=\|z-T z\|,
$$

thus $B_{b} \subseteq B_{z}$ (since $b$ is a common point of $B_{b}$ and $B_{z}$ ) and $T b \in B_{z}$.

Now, if $\|b-T b\|<\|z-T z\|$ then

$$
\|b-z\|=\|z-T z\|>\|b-T b\| \text {. }
$$

This implies that $z \in B_{z}$ but $z \notin B_{b}$, which is impossible from the maximality of $B_{z}$. Thus

$$
\|b-T b\|=\|z-T z\|=: r \quad \forall b \in B_{z} .
$$

Remark. Evidently the above results hold also in the case of a spherically complete ultrametric space.

\section{EXAMPLES}

1. Let $X$ be the space $c_{0}$ over $\mathscr{K}$ with the valuation of $\mathscr{K}$ discrete. It is well known that in this case $c_{0}$ is spherically complete. Let $\pi \in \mathscr{K}$ with 
$0<|\pi|<1$. Suppose $T: c_{0} \rightarrow c_{0}$ is the mapping defined by

$$
T\left(x_{1}, x_{2}, x_{3}, \ldots\right)=\left(\pi, x_{1}, x_{2}, x_{2}, \ldots\right) .
$$

Clearly $\|T x-T y\| \leq\|x-y\|$, but $T$ has no fixed point in $c_{0}$.

2. Let $X$ be the space $c_{0}$ over $\mathscr{K}$ with the valuation of $\mathscr{K}$ dense. As it is known, $c_{0}$ in this case is not spherically complete. Suppose $T$ is the mapping $T: c_{0} \rightarrow c_{0}$ defined by

$$
T\left(x_{1}, x_{2}, x_{3}, \ldots\right)=\left(1, s_{1} x_{1}, s_{2} x_{2}, \ldots, s_{n} x_{n}, \ldots\right),
$$

where $\left\{s_{n}\right\}$ is a sequence in $\mathscr{K}$ with $\left|s_{n}\right|<1$ for all $n \in \mathbb{N}, \lim _{n \rightarrow \infty}\left|s_{n}\right|=1$, and $\lim _{n \rightarrow \infty} \prod_{i=1}^{n}\left|s_{i}\right| \neq 0$. The mapping $T$ is clearly a contraction but it has no fixed points.

3. Let $X$ be the space $c_{0}$ with $\mathscr{K}$ nonspherically complete. As is known, $c_{0}$ in this case is reflexive. The mapping of example 1 maps the unit sphere of $c_{0}$ into itself but does not have a fixed point.

So in non-Archimedean spaces, a nonexpansive mapping on a reflexive space, which maps a convex closed and bounded set into itself, does not necessarily have a fixed point.

\section{REFERENCES}

1. D. E. Alspach, A fixed point free nonexpansive mapping, Proc. Amer. Math. Soc. 82 (1981), 423-424.

2. J. B. Baillon and R. Schöneberg, Asymptotic normal structure and fixed points of nonexpansive mappings, Proc. Amer. Math. Soc. 81 (1981), 257-264.

3. W. A. Kirk, A fixed point theorem for mappings which do not increase distance, Amer. Math. Monthly 72 (1965), 1004-1006.

4. A. C. M. van Rooij, Non-Archimedean functional analysis, Marcel Dekker, New York, 1978.

Department of Mathematics, University of Ioannina, Ioannina, Greece

E-mail address, C. Petalas: Petalas@grioanun

E-mail address, T. Vidalis: Vidalis@grioanun 